\title{
Trombosis de vena cava inferior y endocarditis en un paciente prematuro. Caso clínico
}

\author{
A. Parra • N. Valencia • A. Uribe
}

\begin{abstract}
Inferior vena cava thrombosis and endocarditis in a premature patient - Case report

Thrombosis of the inferior vena cava represents a significant percentage of all venous thrombosis that take place during the neonatal period, generally associated with risk factors such as the use of central venous catheter. The incidence of bacterial endocarditis in preterm infants is low. Objectives: To characterize the case of a preterm neonate with both conditions and to detail the disease changing spectrum in the neonatal population and its therapeutic possibilities. Case report: Premature newborn, $31+5$ weeks of gestation who presented Enterococcus faecalis bacteremia, developed progressive thrombosis of the inferior vena cava and right atrium secondary to the use of umbilical venous catheter, with subsequent diagnosis of endocarditis. He was treated with anticoagulation with subcutaneous low molecular weight heparin. Given a favorable evolution, it was decided to continue the anticoagulation therapy for 4-6 weeks, and at the time of discharge, aspirin treatment was given. Conclusions: The preterm infant with infective endocarditis and intracardiac thrombus presents an interesting management dilemma. Treatment should be individualized according to the clinical evolution and safety profile of thrombolytic and/or anticoagulant agents. Availability and advantages of low molecular weight heparin have led to its use as an alternative treatment in neonates and infants with deep venous thrombosis. (Key words: Neonatal bacteremia, central venous catheter, endocarditis, thrombosis).

Rev Chil Pediatr 2014; 85 (6): 708-713
\end{abstract}

\section{RESUMEN}

La trombosis de la vena cava inferior corresponde a un porcentaje importante de las trombosis venosas en la etapa neonatal, generalmente asociado a factores de riesgo como el uso de catéter venoso central. La incidencia de endocarditis bacteriana en recién nacidos prematuros es baja. Objetivos: Caracterizar el caso de un neonato pretérmino en que se asociaron ambas patologías y detallar el espectro cambiante de esta enfermedad en la población neonatal y sus posibilidades terapéuticas. Caso clínico: Recién nacido prematuro de $31+5$ semanas de gestación, que presentó bacteriemia por Enterococo faecalis, evolucionó con trombosis progresiva de la vena cava inferior y aurícula derecha secundaria al uso de catéter venoso umbilical, con posterior diagnóstico de endocarditis. Se manejó con anticoagulación con heparina de bajo peso molecular en forma subcutánea,

Recibido el 10 de octubre de 2012. Última versión aceptada el 01 de octubre de 2014.

Andrea Parra Buitrago ( $\varangle)$, Natalia Andrea Valencia Zuluaga

Pediatra,Clínica Universitaria Bolivariana, Hospital Pablo Tobón Uribe, Docente Universidad Pontificia Bolivariana.

E-mail: andreaparrab@gmail.com

Andrés Felipe Uribe Murillo, MD

Pediatra, Líder científico, Unidad de Neonatología Clínica Universitaria Bolivariana. Docente Universidad Pontificia Bolivariana. 
dada evolución favorable, se decidió continuar manejo médico con terapia anticoagulante por 4-6 semanas, y al alta manejo con aspirina. Conclusiones: El neonato pretérmino con endocarditis infecciosa y trombo intracardiaco presentan un dilema interesante de manejo, por lo cual se debe individualizar el tratamiento según la evolución clínica y el perfil de seguridad de los agentes trombolíticos y/o anticoagulantes. La disponibilidad y las ventajas de la heparina de bajo peso molecular ha dado lugar a su uso como una alternativa de tratamiento en neonatos y niños con trombosis venosa profunda.

(Palabras clave: MeSH: bacteriemia neonatal, catéter venoso central, endocarditis, trombosis).

Rev Chil Pediatr 2014; 85 (6): 708-713

\section{Introducción}

El período neonatal es uno de los períodos más frecuentes para el desarrollo de complicaciones trombóticas. La incidencia de trombosis neonatal ha sido estimada en un 5,1 por 100.000 nacidos vivos, o 2,4 por cada 1.000 recién nacidos ingresados a unidades de cuidados intensivos neonatales. En diferentes estudios, el $89 \%$ de la formación de trombos fue relacionada con la presencia de catéter venoso central, de los cuales $29 \%$ se asoció con infección sistémica ${ }^{1,2}$.

El número de niños que reciben tratamiento anticoagulante es cada vez mayor. Los eventos tromboembólicos se asocian con un significativo riesgo de morbilidad y mortalidad ${ }^{3}$.

Para el diagnóstico de la enfermedad es importante la confirmación ecográfica, pero como el tratamiento quirúrgico para trombos intracardíacos en los recién nacidos se asocia con alta mortalidad, se prefiere el manejo médico, pudiendo ser la única modalidad viable de tratamiento en algunos casos. Los últimos estudios han evaluado la anticoagulación en los niños desde el punto de vista de las complicaciones de los agentes antitrombóticos disponibles ${ }^{4,5}$.

Entre los tratamientos disponibles esta la estreptoquinasa, uroquinasa y activador de plasminógeno tisular recombinante (r-TPA), pero la Heparina de bajo peso molucular (HBPM), aunque no es un trombolítico sino un anticoagulante, puede ser una opción terapéutica para la trombosis en neonatos de alto riesgo. Su uso pretende evitar el crecimiento del coágulo/trombo, teniendo en cuenta especialmente la facilidad de su administración por vía subcutánea, la farmacocinética predecible y menor incidencia de efectos adversos, tales como complicaciones hemorrágicas ${ }^{6}$.

El propósito del artículo es: a) Describir el caso de un neonato pretérmino con una trombosis venosa profunda (TVP) grave asociada al uso de catéter venoso central (CVC) y sospecha de endocarditis que responde en forma exitosa al tratamiento anticoagulante; b) Detallar el espectro cambiante de esta enfermedad en la población neonatal y sus posibilidades terapéuticas.

\section{Caso clínico}

Recién nacido femenino, fruto de embarazo gemelar monocorial biamniótico, nacido por cesárea por madre con preeclampsia, a las $31+5$ semanas de gestación, con peso: $1.010 \mathrm{~g}$. Apgar 6/8. Nació con dificultad respiratoria leve, y quejido, se realizó radiografía de tórax sugerente de enfermedad de membrana hialina grado 2, por lo que se decidió intubar para administrar surfactante, con mejoría radiológica y posterior extubación. Se instalaron catéteres umbilicales sin complicaciones inmediatas.

A los 10 días de vida, se auscultó soplo cardíaco por lo que se indicó ecocardiografía, encontrándose trombo en vena cava inferior (VCI) y entrada de aurícula derecha (AD) (figura 1). Dado que se sospechó que dicho trombo se podía asociar a presencia de catéter umbilical, se inició anticoagulación con HBPM subcutánea.

Se le realizó nueva ecocardiografía a los 14 días de vida en la cual se observó foramen oval (FO) permeable, trombo en VCI y en $\mathrm{AD}$ que pasaba por el FO hacia la aurícula izquierda (AI), con alto riesgo de embolización (figura 
2). Se evaluó con equipo médico cardiovascular para terapia trombolítica con r-TPA. Esta terapia no se pudo realizar dado que en los exámenes de control se observó aumento de los reactantes de fase aguda (tabla 1), sospechándose endocarditis.

Por este motivo se decidió policultivar e iniciar antibioticoterapia empírica con cefalosporinas de tercera generación. Con hemocultivos positivos y líquido cefalorraquídeo (LCR) anormal, se diagnosticó sepsis tardía nosocomial con compromiso meníngeo. Por aislamiento de Enterococcus faecalis sensible a ampicilina en hemocultivo, se cambió terapia por ampicilina más gentamicina para completar tratamiento por 4-6 semanas.

Se realizó al mes de vida ecocardiografía de control, destacando disminución del tamaño del trombo (figura 3), con hemocultivos de

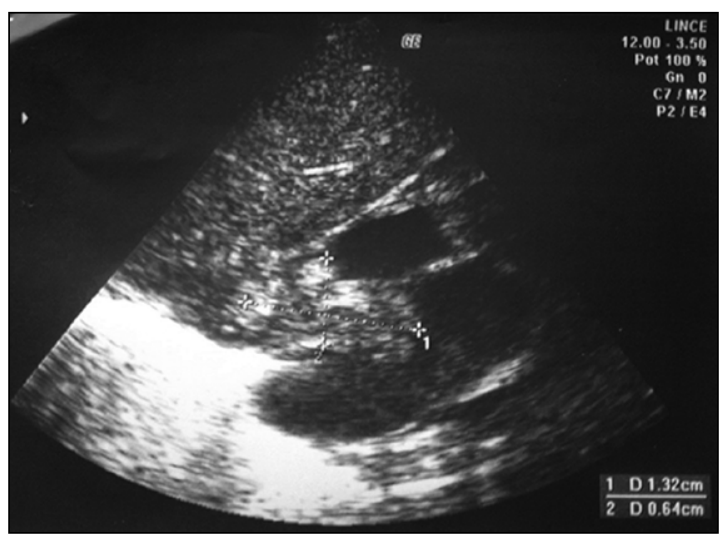

Figura 2. Ecocardiografia: A los 14 días de vida se observa foramen oval permeable, trombo en vena cava inferior y en auricula derecha (AD) que pasa por el foramen oval hacia la aurícula izquierda (AI), con alto riesgo de embolizar. Trombo en vena cava inferior, $\mathrm{AD}$ que pasa por el foramen oval hacia la $\mathrm{AI}(13,2 * 6,4 \mathrm{~mm})$.

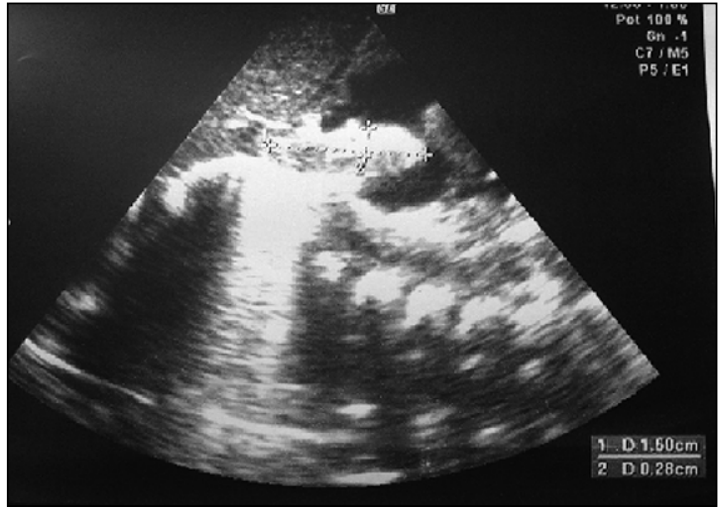

Figura 1. Ecocardiografia: A los 10 días de vida, visión subcostal que demuestra trombo de $15 \mathrm{~mm}$ por $2,8 \mathrm{~mm}$ que se extiende desde la vena cava inferior hasta la entrada de AD. Trombo en vena cava inferior y entrada de AD. $15 * 2,8 \mathrm{~mm}$ sin otros hallazgos. (AD: auricula derecha).

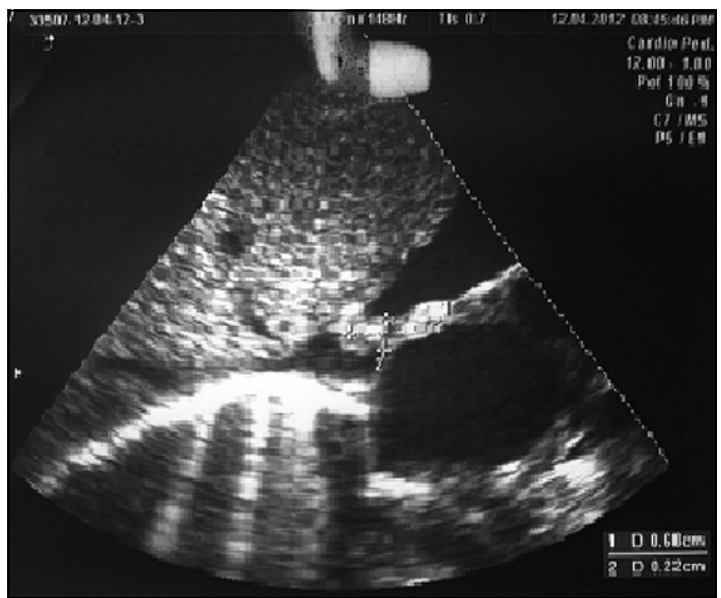

Figura 3. Ecocardiografia al mes de vida con disminución del tamaño del trombo, foramen oval permeable, persiste imagen de trombo en vena cava inferior, atrio derecho hasta atrio izquierdo pero de menor tamaño mide $6,8 * 2,2 \mathrm{~mm}$.

Tabla 1. Evolución de exámenes de laboratorio durante la estadia hospitalaria

\begin{tabular}{|cccccc|}
\hline Edad (días) & Hb (g/d) & Hto (\%) & Leucocitos & Plaquetas & PCR (mg/dl) \\
\hline 1 día & 16,3 & 48 & 9.080 & 175.800 & 0,4 \\
\hline 8 días & 13,6 & 39 & 9.270 & 207.200 & 0,6 \\
\hline 16 días & 10 & 29,5 & 31.770 & 352.000 & 6 \\
\hline 2 días & 13,04 & 38,5 & 26.170 & 358.000 & 14 \\
\hline 25 días & 12,4 & 36,9 & 13.850 & 340.000 & 11,2 \\
\hline 30 días & 13,09 & 37 & 11.400 & 256.000 & 1,3 \\
\hline
\end{tabular}

Hb: Hemoglobina; Hto: hematocrito; PCR: proteína $\mathrm{C}$ reactiva. 
control negativos y descenso de PCR, por lo que se descartó posible trombolisis decidiendo continuar manejo médico con terapia anticoagulante por 4-6 semanas, y al alta manejo con aspirina.

\section{Discusión}

Los recién nacidos y especialmente los prematuros están en riesgo de trombosis debido a las concentraciones plasmáticas de procoagulante, anticoagulante, y factores fibrinolíticos disminuidos en comparación con niños mayores $^{7}$. El riesgo aumenta por la presencia de un catéter venoso central permanente, o por ciertas condiciones médicas como la prematuridad, la sepsis, asfixia perinatal, la cirugía, cardiopatías congénitas o cáncer ${ }^{8}$.

Aparte de la presencia de un catéter venoso umbilical por varios días, que fue el factor inicial para la aparición de un trombo en vena cava inferior y aurícula derecha en el paciente del caso clínico, la septicemia fue un factor adicional de riesgo de trombosis y morbilidad por la presencia de endocarditis infecciosa.

Desde la década de 1960, la tasa de endocarditis infecciosa se ha incrementado debido al aumento de la supervivencia de los niños con cardiopatías congénitas, y un mayor uso de $\mathrm{CVC}^{9-11}$.

Una amplia variedad de microrganismos pueden ocasionar endocarditis infecciosa, un estudio de cohorte prospectivo en 2.781 pacientes, identificó Staphylococcus aureus en un $31 \%$ de los casos y el Streptococcus sp con un $17 \%$ y diferentes variedades de Enterococcus en un $11 \%{ }^{12,13}$.

Una lesión en el endotelio es un potente inductor de trombogénesis, ocasionando que las bacterias se puedan adherir y formar una vegetación. La endocarditis neonatal ocurre con frecuencia en el lado derecho del corazón asociada a la interrupción del endocardio o tejido endotelial valvular producido por un traumatismo con los catéteres, la combinación de daño endotelial y bacteriemia es crítica para la inducción de endocarditis infecciosa ${ }^{14}$. Los cultivos de sangre se deben realizar en todos los pacientes con sospecha diagnóstica al igual que la ecocardiografía. Y el tratamiento antibiótico se debe instaurar ante la sospecha diagnostica, el fracaso del tratamiento médico en pacientes con vegetaciones persistentes pueden hacerlo pensar en una intervención quirúrgica, sopesando los riesgos vs los beneficios $^{15,16}$.

Cuando existe trombosis de vena cava inferior o trombosis de la aurícula derecha, como fue el caso de este paciente, una revisión sistemática sugiere que una trombosis de la aurícula derecha pequeña menor de $2 \mathrm{~cm}$ en cada dimensión e inmóvil pueden ser tratado de manera conservadora, porque la trombectomia o terapia trombolítica conlleva un riesgo significativo de mortalidad quirúrgica y sangrado ${ }^{17,18}$.

Un reporte de caso ${ }^{16}$, mostró 31 ciclos de terapia trombolítica realizada en 23 neonatos con trombosis de VCI, la infusión continua de trombolíticos $(\mathrm{n}=26)$ o la aplicación repetida de trombolíticos a corto plazo $(n=4)$ dio como resultado la recanalización completa y parcial de la vena cava inferior en 12 casos y 3 casos respectivamente, pero se fracasó en 8 . La terapia con r-tPA fue más eficaz que la terapia sistémica con uroquinasa ( 3 de 6 frente a 3 de 10), mientras que la trombolisis con estreptoquinasa fue ineficaz en todos los casos, reportándose complicaciones en $3 / 23$ recién nacidos, como hemorragias cerebrales, intracerebrales y subependimarias, consideradas de mayor riesgo en recién nacidos muy prematuros ${ }^{19}$.

Cabe resaltar que estos bebés prematuros a menudo son de bajo peso al nacer, por lo que la opción del tratamiento quirúrgico técnicamente es más difícil, además de gran morbilidad y mortalidad, por lo cual la terapia médica es casi la única opción de tratamiento para estos niños, pero la preferencia de tratamiento entre los agentes trombolíticos varía entre los diferentes centros.

La disponibilidad y las ventajas de uso de la HBPM han dado lugar a su uso como una alternativa de tratamiento en neonatos y niños con trombosis venosa profunda. Se hace especial énfasis en que la HBPM no es trombolítico y en el paciente de nuestro caso se usó como anticoagulante para evitar el crecimiento del trombo. Las recomendaciones actuales 
de la literatura son el manejo con heparina no fraccionada o HBPM por aproximadamente 6 meses, y el catéter debe ser retirado 3 a 5 días posterior al inicio de la anticoagulación, como ocurrió en este caso, siendo importante aclarar que no se pudo realizar monitorización de dicho tratamiento ya que no contamos con medición de actividad anti-factor Xa. El tratamiento precoz se recomienda debido al riesgo inherente de la enfermedad tromboembólica, el activador del plasminógeno tisular puede desempeñar un papel importante cuando la terapia estándar ha fallado dado por aumento del tamaño del trombo durante la terapia y el empeoramiento de la función valvular o ventricular, por medio de ecocardiografía, además debe cumplir con un valor mínimo permitido de plaquetas, fibrinógeno y hemoglobina, hematocrito para asegurar su utilización ${ }^{20}$.

\section{Conclusión}

Los recién nacidos pretérmino a menudo experimentan episodios transitorios de bacteriemia, en el cuidado intensivo neonatal, por el trauma en la piel y mucosas, aspiración endotraqueal vigorosa, nutrición parenteral, CVC, los cuales son también factores adicionales de riesgo para la trombosis, la combinación de daño endotelial es un potente inductor de trombogénesis y la bacteriemia es crítica para la inducción de endocarditis infecciosa.

Cuando existe trombosis en la superficie interna del corazón y endocardio la HBPM puede ser una opción terapéutica en neonatos de alto riesgo, especialmente teniendo en cuenta la facilidad de su administración por vía subcutánea, farmacocinética predecible y menor incidencia de efectos adversos, porque la trombéctomia o terapia trombolítica conlleva un riesgo significativo de mortalidad quirúrgica y/o sangrado.

Debido a que la enfermedad trombótica puede causar una morbilidad significativamente grave, es importante definir según el riesgo beneficio, la mejor opción terapéutica para cada tipo de paciente, de acuerdo a las posibilidades terapéuticas antitrómboticas y anticoagulantes para la población neonatal.
Potenciales conflictos de interés: Este trabajo cumple con los requisitos sobre consentimiento/asentimiento informado, comité de ética, financiamiento, estudios animales y sobre la ausencia de conflictos de intereses según corresponda.

\section{Referencias}

1.- Monagle P, Newall F: Anticoagulation in children. Thromb Res 2012; 130 (2): 142-6.

2.- Andrew ME, Monagle P, deVeber G, Chan AK: Thromboembolic disease and antithrombotic therapy in newborns. Hematology Am SocHematol Educ Program 2001; 358-74.

3.- Payne JH: Aspects of anticoagulation in children. Br J Haematol 2010; 150 (3): 259-77.

4.- Chan VH, Monagle P, Massicotte P, Chan AK: Novel paediatric anticoagulants: a review of the current literature. Blood Coagul Fibrinolysis 2010; 21 (2): 144-51.

5.- Newall F, Ignjatovic V, Johnston L, et al: Age is a determinant factor for measures of concentration and effect in children requiring unfractionated heparin. Thromb Haemost 2010; 103 (5): 1085-90.

6.- Häusler M, Hübner D, Delhaas T, Mühler EG: Long term complications of inferior vena cava thrombosis. Arch Dis Child 2001; 85 (3): 228-33.

7.- Monagle P, Chan AK, Goldenberg NA, et al: Antithrombotic Therapy and Prevention of Thrombosis, 9th ed: American College of Chest Physicians Evidence-Based Clinical Practice Guidelines. Chest 2012; 141 (2 Suppl): e737S-801S

8.- Long E, Pitfield AF, Kissoon N: Anticoagulation Therapy: Indications, monitoring, and complications. Pediatr Emerg Care 2011; 27 (1): 55-61.

9.- Day MD, Gauvreau K, Shulman S, Newburger JW: Characteristics of children hospitalized with infective endocarditis. Circulation 2009; 119 (6): 865-70.

10.- Marom D, Levy I, Gutwein O, Birk E, Ashkenazi S: Healthcare-associated versus community-associated infective endocarditis in children. Pediatr Infect Dis J 2011; 30: 585-8.

11.- García-Teresa MA, Casado-Flores J, Delgado Domínguez $M A$, et al: Infectious complications of percutaneous central venous catheterization in pediatric patients: a Spanish multicenter study. Intensive Care Med 2007; 33: 466-76.

12.- Fowler VG Jr, Miro JM, Hoen B, et al: Staphylococcus aureus endocarditis: a consequence of medical progress. JAMA 2005; 293 (24): 3012-21.

13.- Murdoch DR, Corey GR, Hoen B, et al: Clinical presentation, etiology, and outcome of infective endocarditis 
in the 21st century: the International Collaboration on Endocarditis-Prospective Cohort Study. Arch Intern Med 2009; 169 (5): 463-73.

14.- Ferrieri P, Gewitz MH, Gerber MA, et al: Unique features of infective endocarditis in childhood. Pediatrics 2002; 109 (5): 931-43.

15.- Habib G, Hoen B, Tornos P, et al: Guidelines. Sophia Antipolis, France: European Society of Cardiology; 2009.

16.- Hoen B, Duval X: Infective endocarditis. N Engl J Med 2013; 368: 1425-33.

17.- Ferrari F, Vagnarelli F, Gargano $G$, et al: Early intracardiac thrombosis in preterm infants and thrombolysis with recombinant tissue type plasminogen activator.
Arch Dis Child Fetal Neonatal Ed 2001; 85 (1): F66-9.

18.- Yang JY, Williams S, Brandão LR, Chan AK: Neonatal and childhood right atrial thrombosis: recognition and a risk-stratified treatment approach. Blood Coagul Fibrinolysis 2010; 21 (4): 301-7.

19.- Häusler M, Hübner D, Hörnchen H, Mühler EG, Merz $U$ : Successful Thrombolysis of Inferior Vena Cava Thrombosis in a Preterm Neonate. Clin Pediatr (Phila) 2001; 40 (2): 105-8.

20.- Anderson B, Urs P, Tudehope D, Ward C: The use of recombinant tissue plasminogen activator in the management of infective intracardiac thrombi in pre-term infants with thrombocytopaenia. J Paediatr Child Health 2009; 45 (10): 598-601. 